\title{
Nasopalpebral lipoma-coloboma syndrome
}

INSERM

\section{Source}

INSERM. (1999). Orphanet: an online rare disease and orphan drug data base. Nasopalpebral lipoma-coloboma syndrome. ORPHA:2399

Nasopalpebral lipoma-coloboma-telecanthus syndrome is characterized by nasopalpebral lipomas, bilateral lid coloboma, and telecanthus. 